\title{
Cetrorelix preserves follicular viability in Cyclophosphamid-induced ovarian toxicity
}

\author{
Mohammadnejad $\mathrm{D}^{1}$, Tayefi-nasrabadi $\mathbf{H}^{2}$, Naghibi $\mathbf{M}^{2}$, Abedelahi $\mathrm{A}^{2 *}$ \\ ${ }^{1}$ Drug Applied Research Center, Tabriz University of Medical Sciences, Tabriz, Iran \\ ${ }^{2}$ Department of Anatomical Sciences, Tabriz University of Medical Sciences, Tabriz, Iran \\ *Corresponding author: Abedelahi A, Tabriz University of Medical Sciences, Tabriz, Iran, Email: abedelahia@yahoo.com, \\ Zip code: 5714783734 , Tel: +98 4133342086
}

\begin{abstract}
Background \& Aims: Anticancer drugs are known to have great impact on spermatogenesis process and germinal epithelium. The present study is to investigate the preventive effect of Cetrorelix (GnRH antagonist) on Cyclophosphamide- induced spermatogenic defect.

Methods and Materials: Fifty adult female mice aging 6-8 weeks (30 \pm 4 gr) were divided into 3 groups as: Control, Experimental 1 and Experimental 2. The animals in Experimental 1 group received Cyclophosphamide $(2.5 \mathrm{mg} / \mathrm{kg}$, ip) for 5 days and in Experimental 2 group received Cetrorelix $(0.25 \mathrm{mg} / \mathrm{kg}$, ip) one week before Cyclophosphamide treatment and continued for 3 weeks. The mice in all groups were sacrificed 35 days after the last injection. Ovarian follicles were isolated mechanically and the viability was studied by trypan blue staining.

Results: Ovarian follicles of animals in Experimental 2group, retained higher percentage of normal morphology $(\mathrm{P}<0.001)$ than which in Experimental 1 group and their condition were similar to control group.

Conclusion: These results indicate that the Cetrorelix administration before cancer treatment may protect ovarian tissue against side effects of cisplatin.
\end{abstract}

Keywords: Ovarian follicles, Viability, GnRH antagonist, Cetrorelix

Received 7 May 2015, accepted for publication 29 July 2015

\section{Introduction}

Over the last decades attempts have been made to minimize the toxic effects of chemotherapy on the ovarian tissue (1). The alkylating drug Cyclophosphamide (CP) is a commonly used chemotherapeutic agent for cancer treatment. A number of reports described the adverse effects of Cyclophosphamide on fertility in human and animals (25).Chemotherapeutic agents are mostly effecting rapidly dividing cells such as cancer cells and gonadal cells. Unfortunately, these agents cannot distinguish the difference between gonadal-from cancer-cells which leads to chemotherapeutic agents induced destruction of gonadal cells during and results in premature ovarian failure (6-7).Alternative methods have been developed to decrease the adverse effects of chemotherapy on ovarian functions. It is known that oogenesis is reduced by hypothalamic- pituitary axis (FSH, LH) suppression. This can be achieved by using antagonist of GnRH such as Cetrorelix. Recently, we reported that Cetrorelix administration protect seminiferous tubules structure from side effects of chemotherapeutic agents (8) but the preventive effect of Cetrorelix on oogenesis has poorly been studied. Therefore, the present study was designed to investigate the protective effect of Cetrorelix on the viability of ovarian follicles following treatment with Cyclophosphamide.

\section{Materials and Methods \\ Chemicals:}

All chemicals and media used in the present study were purchased from Sigma-Aldrich Co. (HamburgGermany) unless otherwise indicated. 


\section{Animals and Ovarian Tissue Preparation:}

Five to six week-old Balb/c female mice $(n=15)$ were housed in light and temperature controlled condition (12-h light/12-h dark, $22-24^{\circ} \mathrm{C}$ and $55 \%$ humidity).Food and water were freely available at all times. All the animals were handled in accordance with the guidelines of Tabriz University of Medical Sciences Committee for Animal Care.

In experimental 1 group, mice received $50 \mathrm{mg} / \mathrm{kg}$ daily Cyclophosphamide intraperitonally for five days. In experimental 2 groups the mice in addition to Cyclophosphamide received Cetrorelix subcutaneously $0.25 \mathrm{mg} / \mathrm{kg} 3$ times a week. Cetrorelix application started first week prior to Cyclophosphamide treatment and continued for three weeks. In all groups, mice were sacrificed by cervical dislocation 35 days after last injection and their ovaries were dissected free of fat and mesentery.

\section{Evaluation of the Ovarian Follicular Viability:}

Ovaries in control and each experimental groups $(\mathrm{n}=$ 5 for each group) were placed in $100 \mu 1$ microdroplets medium as described earlier. Ovarian follicles in different stages were mechanically isolated from the ovaries using 29-gauge needles under a stereomicroscope (SZ-STS, Olympus, Tokyo, Japan)and were transferred to new microdroplets $(20 \mu \mathrm{l})$ of medium and covered with mineral oil. All ovarian follicles categorized to the primordial follicle with one squamous cells layer with $30-50 \mu \mathrm{m}$ diameter, primary follicle with one cuboidal granulosa cells layer with 50-90 $\mu \mathrm{m}$ diameter and secondary follicle with two or three layer of granulosa cell and 100-150 $\mu \mathrm{m}$ diameter. For each group, only follicles containing layers of membraneenclosed granulosa cells with a centrally located oocyte were examined. They were stained using $0.4 \%$ trypan blue and examined under an inverted microscope (Olympus, CKX41, Japan). The follicles were classified as intact (were not stained and the oocyte and the surrounding granulosa cells were clear) or degenerated (follicles stained blue) (Fig1.A-D).

\section{Statistical analysis:}

Statistical analysis was done with SPSS 16.0 software. The survival rates of follicles in vitrified, nonvitrified and toxicity tested groups were compared by one-way analysis of variance (ANOVA) and Tukey's test. A $P<0.05$ was considered to be statistically significant.

\section{Results}

Percentages of viable ovarian follicles derived from control and experimental groups tested ovaries after trypan blue staining is presented in Table 1.

Table 1: The viability of ovarian follicles in various stages isolated from control and experimental groups

\begin{tabular}{|c|c|c|c|c|c|c|c|c|c|c|}
\hline \multirow[t]{2}{*}{ Group } & \multirow{2}{*}{$\begin{array}{l}\text { Total } \\
\text { number } \\
\text { of } \\
\text { follicles }\end{array}$} & \multicolumn{3}{|c|}{$\begin{array}{l}\text { Number of primordial } \\
\text { follicles }(\%)\end{array}$} & \multicolumn{2}{|c|}{$\begin{array}{l}\text { Number of primary } \\
\text { follicles }(\%)\end{array}$} & \multicolumn{4}{|c|}{$\begin{array}{l}\text { Number of secondary follicles } \\
\qquad(\%)\end{array}$} \\
\hline & & Total & Int & Deg & Total & Int & Deg & Total & Int & Deg \\
\hline Control & 124 & 44 & $42(95)$ & $2(5)$ & 42 & $40(95)$ & $2(5)$ & 38 & $36(95)$ & $2(5)$ \\
\hline Exp 1 & 138 & 47 & $37(79)^{\mathrm{a}}$ & $10(21)$ & 51 & $38(75)^{\mathrm{a}}$ & $13(25)$ & 40 & $28(70)^{\mathrm{a}}$ & $12(30)$ \\
\hline Exp2 & 144 & 46 & $42(91)$ & $4(9)$ & 57 & $52(91)$ & $5(9)$ & 41 & 37 (90) & $4(10)$ \\
\hline
\end{tabular}

${ }^{\mathrm{a}} \mathrm{P}<0.001$ versus control and Exp2 group, intact (Int) and degenerated (Deg) 


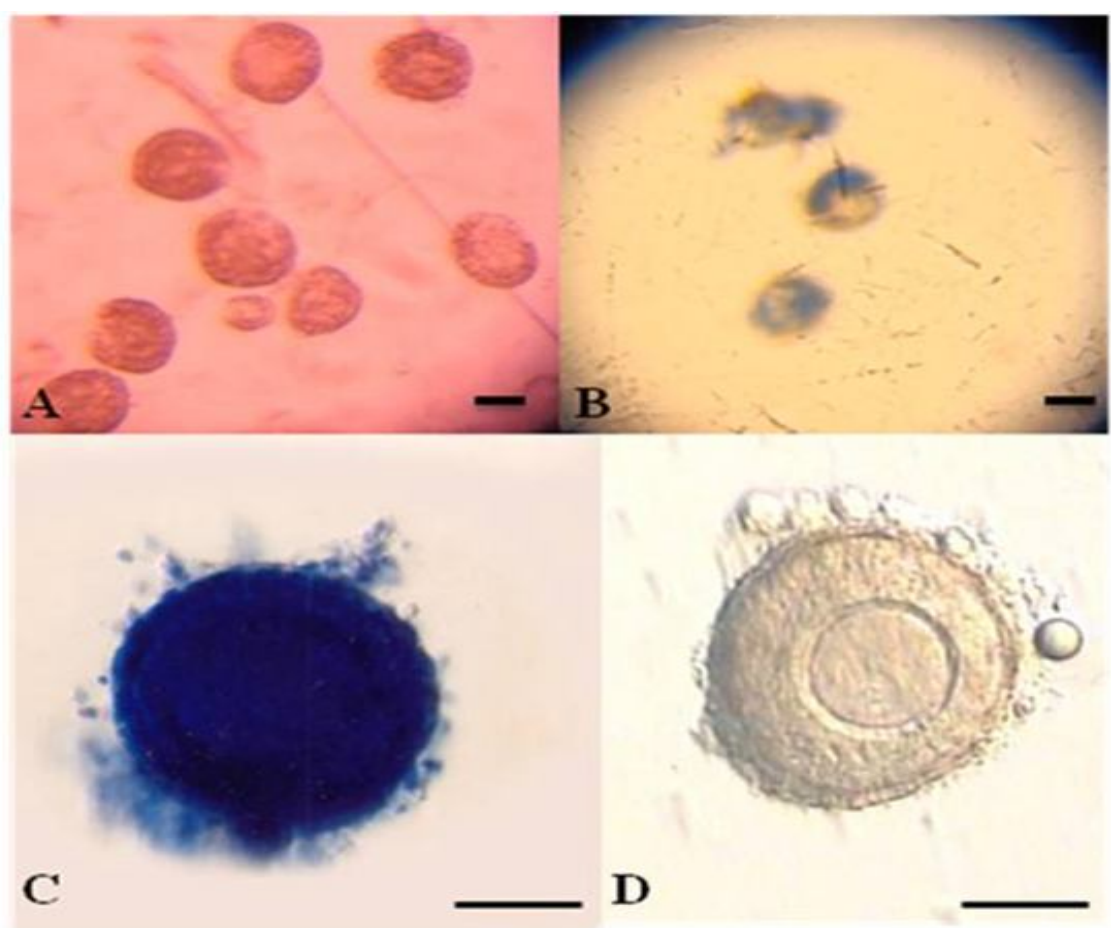

Figure 1: Trypan Blue staining for viability of isolated secondary follicles from vitrified ovarian tissues. (A);

Primordial and Primary follicles considered as live follicles after trypan blue staining. (B); Primordial and Primary follicles considered as dead or degenerated follicles with blue staining. (C); Secondary follicle with blue oocyte and follicular cells considered as dead follicle. (D); Secondary follicle as live follicles after trypan blue staining. Scale bar $=50 \mu \mathrm{m}$

Ovarian follicles were mechanically isolated from control and experimental 1, 2and approximately 45primordial, 50 primary and 39 secondary follicles in each group were examined for their viability. The percentage of viability of ovarian folliclesin different stages derived from control and experimental groups were $95.14 \%, 74.41 \%$, and $90.92 \%$, respectively (Table 1).Exposure to $2.5 \mathrm{mg} / \mathrm{kg}$ of Cyclophosphamide (Exp1) in a main significantly decreases $74 \%$ in differential stages of ovarian follicles compared to untreated controls $(\mathrm{P}<0.001)$.

Pretreatment with Cetrorelix (Exp2) resulted in a significantly higher percentage of ovarian follicle in different stages respectively than Cyclophosphamide treatment $($ Exp1) group $(\mathrm{P}<0.001)$; primordial follicles (91\% vs78\%), primary follicles (91\% vs $74 \%)$ and secondary follicles $(90 \%$ vs $70 \%)$. In the present study we could not show any significant differences between control and Cetrorelix treatment group (Exp2) in differential stages of ovarian follicles. A very low rate of degeneration was observed in Exp2 group compared with $\operatorname{Exp} 1$ (9\% vs25\%).

\section{Discussion}

We found that Cyclophosphamide treatment in mice led to impaired ovarian follicular viability. Pretreatment with the GnRH-antagonist such as Cetrorelix protected ovarian follicle viability in different stages of development.

Many chemotherapeutic agents, especially alkylating agents alter fertility in females by effecting granulosa cell proliferation and ovarian hormone (estradiol) secretion (9).After Cyclophosphamide injection, a significant reduction in number of granulosa cells and 
delay in cell cycle progression was observed which can effect cell proliferation (10).Moreover, the secretion of endogenous FSH accelerated after Cyclophosphamide treatment and cell cycle of the granulose have been shortened by FSH that enhances follicular maturation, whereby Cyclophosphamide accelerates follicular recruitment and atresia and diminishes the number of follicles in the ovaries $(11,12)$.

In mice, the follicular development takes 3 to 5 weeks (13), therefore in this study a 5week treatment designed which may affect all follicular stages. Finding of present study showed significantly decreases in the number of ovarian follicles after treatment by Cyclophosphamide, these findings are in accordance with findings of Ataya et al. study (14).

Chemotherapy is known to induce apoptosis in granulosa cells by induction of oxidative stress leading to follicular degeneration (15), in this study, follicular degeneration in different stages of development was also observed.

Our results suggested that 35-days treatment of female mice with Cyclophosphamide can decrease ovarian folliculogenesis and steroidogenesis. Therefore, co-administration of GnRH in this experiment was maintained folliculogenesis at control.

GnRH antagonists such as Cetrorelixdirectly bind to $\mathrm{GnRH}$ receptors in granulosa cells of ovarian follicles (16). GnRH receptor expression differs among follicles in different stages (17). Therefore, the least damage was observed in the primordial follicle compared to other stages due to their special characteristics (their small size, a low number of granulosa cells around the small oocyte) after treatment.

The results of present study indicated that the secondary follicles seem vulnerable to Cyclophosphamide-induced damages and the higher rate of degenerative follicles observed in secondary follicles of ovarian tissue that induce decrease of fertility rate and implantation, therefore increase fetal malformations. The ovarian tissue treated with Cetrorelix may overcome
Cyclophosphamide-induced damages and return viability of follicles.

Plowchalk et al. reported that Cyclophosphamide functions by DNA alkylation and subsequent disruption of normal cellular processes and it is most cytotoxic to mitotically active cells (18). Therefore, GnRH antagonist treatment reduces the number of degeneration follicles by limiting the amount of DNA damage in developing ovarian follicles.

Since ovarian tissue contains rapidly dividing cells and undergoes the most rapid mitotic activity, and also chemotherapeutic agents are known to damage primarily rapidly dividing cells, therefore, the ovarian tissue mostly is influenced by cytotoxic effects of Cyclophosphamide.

If cells are allowed to "rest period" or not-dividing activity, the oocyte survives the insult of chemotherapy and repair the lethal damage and seems to return to normal conditions (19).It is assumed that the cells repair the damage during the resting period.

We have demonstrated that Cetrorelix can inhibit ovarian mitotic activity and cause quiescent period, leading to protect side effect of cyclophosphamid. The secretion of FSH and LH seems to be increased after radiotherapy and destructs ovarian tissue structure. Minimizing the secretion of FSH and LH by suppression of hypothalamic-pituitary axisis used to preserve fertility during cancer therapy by reducingoogenesis and follicular proliferation (20) which is reversible after recovery of chemotherapy leading to $\mathrm{FSH}$ and $\mathrm{LH}$ secretion and improves ovarian oogenesis and fertility.

In conclusion our finding indicate that co-treatment of Cetrorelix with Cyclophosphamidcould protect different stages of ovarian follicles from adverse effect of chemotherapy.

\section{Acknowledgments}

We would like to thank Dr. Rahim Beheshti for technical assistance. This work was supported by a grant from Tabriz University of Medical Sciences. 


\section{Conflicts of Interest:}

The authors declare no conflict of interest.

\section{References}

1. Meirow D, Assad G, Dor J, Rabinovici J. The GnRH antagonist Cetrorelix reduces Cyclophosphamide-induced ovarian follicular destruction in mice. Hum Reprod. 2004; 19: $1294-9$.

2. Arora P, Palson DW. Diagnosis and management of premature ovarian failure. Obstet Gynaecol. 2011; 13: 6772.

3. Comish PB, Drumond AL, Kinnell HL, Anderson RA, Matin A, Meistrich ML, et al. Fetal cyclophosphamide exposure induces testicular cancer and reduced spermatogenesis and ovarian follicle numbers in mice. PLoS ONE. 2014; 9(4): e93311.

4. Oh MS, Chang MS, Park W, Kim DR, Bae H, Huh Y, et al. Yukmijihwang-tang protects against cyclophosphamide-induced reproductive toxicity. Reprod Toxicol. 2007; 24(3-4): 365-70.

5. Fleischer RT, Vollenhoven BJ, Weston GC. The effects of chemotherapy and radiotherapy on fertility in premenopausal women. Obstet Gynecol Surv. 2011; 66: 248-54.

6. Howell S, Shalet S. Gonadal damage from chemotherapy and radiotherapy. Endocrinol Metab Clin North Am. 1998; 27(4): 927-43.

7. Meirow D, Nugent D. The effects of radiotherapy and chemotherapy on female reproduction. Hum Reprod Update. 2001; 7(6): 535-43.

8. Mohammadnejad D, Abedelahi A, Rashtbar M. Protective Role of GnRH Antagonist on Chemotherapy-induced Spermatogenesis Disorder: A Morphological Study. Adv Pharm Bull. 2013; 3(2): 323-8.

9. Sonmezer M, Oktay K. Fertility preservation in female patients. Hum. Reprod. Update. 2004; 10: 251-66.

10. MeynR, Murray D. Cell cycle effects of alkylating agents. In: L. Dethlefsen (ed.), Cell Cycle Effects of Drugs. New York: Pergamon Press, Inc; 1986. P.179

11. Ghosh S, Misro M, Das UB, Maiti R, Debnath JM, Ghosh
D. Effect of human chorionic gonadotrophincoadministration on ovarian steroidogenic and folliculogenic activities in cyclophosphamide treated albino rats. Reprod Toxicol. 2001; 15(2): 221-5.

12. Wang XN, Greenwald GS. Hypophysectomy of the cyclic mouse. II. Effects of follicle-stimulating hormone (FSH) and luteinizing hormone on folliculogenesis, FSH and human chorionic gonadotropin receptors, and steroidogenesis. Biol Reprod. 1993; 48(3): 595-605.

13. Hirshfield AN. Development of follicles in the mammalian ovary. Int Rev Cytol. 1991; 124: 43-101.

14. Ataya KM, Valeriote FA, Ramahi-Ataya AJ. Effect of cyclophosphamide on the immature rat ovary. Cancer Res. 1989; 49(7): 1660-4.

15. Gürgen SG, Erdoğan D, Elmas C, Kaplanoğlu GT, Ozer C. Chemoprotective effect of ascorbic acid, $\alpha$-tocopherol, and selenium on cyclophosphamide-induced toxicity in the rat ovarium. Nutrition. 2013; 29(5): 777-84.

16. Kang SK, Choi K-C, Yang H-S, Leung PCK. Potential role of gonadotrophin-releasing hormone $(\mathrm{GnRH})-\mathrm{I}$ and GnRH-II in the ovary and ovarian cancer. Endocr Relat Cancer. 2003; 10(2): 169-77.

17. Bauer-Dantoin AC, Jameson JL. Gonadotropin-releasing hormone receptor messenger ribonucleic acid expression in the ovary during the rat estrous cycle. Endocrinology. 1995 Oct; 136(10): 4432-8.

18. Plowchalk DR, Mattison DR. Reproductive toxicity of Cyclophosphamide in the C57BL/6N mouse: 1. Effects on ovarian structure and function. Reprod Toxicol 1992; 6: $411-421$.

19. Ataya K, Rao LV, Lawrence E, Kimmel R. Luteinizing hormone-releasing hormone agonist inhibits cyclophosphamide-induced ovarian follicular depletion in rhesus monkeys. Biol Reprod. 1995; 52(2): 365-72.

20. Diedrich K, Diedrich C, Santos E, Zoll C, al-Hasani S, Reissmann T, et al. Suppression of the endogenous luteinizing hormone surge by the gonadotrophin-releasing hormone antagonist Cetrorelix during ovarian stimulation. Hum Reprod. 1994; 9(5): 788-91. 